ARTÍCULO ORIGINAL

\title{
Digeneos parásitos de Cyphocharax platanus (Günther, 1880) (Characiformes: Curimatidae) en el tramo inferior del río Uruguay en la zona de influencia de la represa de Salto Grande, Uruguay
}

\author{
Venzal $\mathrm{JM}^{1^{*}}$, Castro $\mathrm{O}^{2}$, Sosa $\mathrm{N}^{1}$, Félix $\mathrm{ML}^{1}$, Leites $\mathrm{V}^{3}$, Menoni $\mathrm{F}^{4}$, Vigil $\mathrm{JD}^{4}$ \\ 1 Laboratorio de Vectores y Enfermedades Transmitidas, Facultad de Veterinaria, CENUR Litoral Norte, Universidad de \\ la República, Uruguay \\ 2 Departamento de Parasitología Veterinaria, Facultad de Veterinaria, Universidad de la República \\ 3 Área de Gestión Ambiental de CTM Salto Grande, Uruguay \\ 4 Ejercicio profesional privado
}

* Correspondencia: José M. Venzal, Laboratorio de Vectores y enfermedades transmitidas, Facultad de Veterinaria, CENUR Litoral Norte - Salto, Universidad de la República, Rivera 1350, 50000, Salto, Uruguay. E-mail: jvenzal@unorte.edu.uy

Recibido: 18 Octubre 2016. Aceptado: 23 Noviembre 2016. Disponible en línea: 30 Noviembre 2016 Editor: P.M. Beldomenico

RESUMEN. Entre agosto de 2012 y junio de 2013 estudios parasitológicos fueron llevados a cabo en 76 ejemplares de Cyphocharax platanus procedentes del tramo inferior del río Uruguay en la zona de influencia de la represa de Salto Grande. Tres taxones de digeneos fueron identificados: Saccocoelioides spp. (Haploporidae) y Zonocotyle bicaecata (Zonocotylidae) en intestino, y metacercarias de Sphincterodiplostomum musculosum (Diplostomidae) en el interior de los ojos. El 69,7\% de los peces analizados resultaron parasitados por al menos un taxón de digeneo. Saccocoelioides spp. mostro los mayores valores de prevalencia, intensidad media y abundancia media $(43,4 \%, 5,45,2,37$ respectivamente) de infección, en relación a Z. bicaecata ( $28,9 \%$, 1,32 y 0,38 respectivamente) y $S$. musculosum $(13,2 \%, 2,82$ y 0,37 respectivamente). Saccocoelioides spp. y $S$. musculosum mostraron un patrón de distribución agregado, en tanto que Z . bicaecata presentó una distribución aleatoria en la población de hospedadores. No se hallaron diferencias significativas en los índices de infección de ninguno de los taxones de digeneos en relación al sexo de los hospedadores, y sólo Saccocoelioides spp. presentó una asociación significativa con el tamaño, siendo más frecuente en peces pequeños. Los dos parásitos intestinales (Saccocoelioides spp. y Z. bicaecata) no presentaron una asociación significativa entre sus índices de infección. Los registros de Saccocoelioides spp. y Z. bicaecata corresponden a los primeros para este hospedador en el río Uruguay, y el hallazgo de las metacercarias de S. musculosum constituye una nueva relación parásito-hospedador.

SUMMARY. Digenean parasites of Cyphocharax platanus (Günther, 1880) (Characiformes: Curimatidae) from the lower Uruguay River, downstream Salto Grande dam, Uruguay. Seventy six specimens of Cyphocharax platanus were collected downstream Salto Grande dam, lower Uruguay River, Uruguay, between August 2012 and June 2013 and examined for digenean parasites. Three digenean taxa were identified: Saccocoelioides spp. (Haploporidae), Zonocotyle bicaecata (Zonocotylidae) (both adult in intestine) and Sphincterodiplostomum musculosum (Diplostomidae) metacercariae inside eyes. We found that $69.7 \%$ of examined fish were parasitized by at least one digenean taxon. Saccocoelioides spp. showed highest values of prevalence, mean intensity and mean abundance $(43.4 \%, 5.45$, and 2.37 , respectively) of infection throughout the study than $Z$. bicaecata $(28.9 \%, 1.32$ and 0.38 , respectively) and S. musculosum (13.2\%, 2.80 and 0.37 , respectively). Saccocoelioides spp. and S. musculosum showed an aggregated distribution pattern, while Z. bicaecata presented a random distribution in the host population. No significant differences in infection indexes of any digenean taxa in relation to host sex were found, and only Saccocoelioides spp. showed a significant association with host size, being more frequent in small fish. The infection indexes of the two intestinal digeneans (Saccocoelioides spp. and Z. bicaecata) were not significantly associated. These are the first records of Saccocoelioides spp. and Z. bicaecata from C. platanus in the Uruguay River, and the finding of $S$. musculosum metacercariae is a new host-parasite relationship.

Palabras clave: Cyphocharax platanus, parásitos, Digenea, río Uruguay, represa de Salto Grande.

Key words: Cyphocharax platanus, parasites, Digenea, Uruguay river, Salto Grande dam. 


\section{Introducción}

Cyphocharax platanus (Günther, 1880) (Characiformes: Curimatidae) es conocido popularmente como "sabalito" y es una de las aproximadamente 41 especies descriptas para el género Cyphocharax Fowler, 1906 (Vari, 2003; Vari et al., 2012; Melo y Vari, 2014; Wosiacki y Miranda, 2013; Dutra et al., 2016). Este género es uno de los ocho que componen la familia Curimatidae siendo el más diverso y con una amplia distribución en el Neotrópico (Vari, 1992). La dieta de los sabalitos es detritívora, y sus juveniles hasta los 40 - 50 días son de hábitos planctófagos (Corrêa y Noguez Piedras, 2008). Como especies forrajeras, son parte de la dieta de una gran variedad de peces y aves ictiófagas. Constituyen una excelente presa para los grandes predadores de los ríos como tarariras, dorados y surubíes, propios de aguas abiertas (Azpelicueta y Braga, 1991). Cyphocharax platanus habita diferentes ecosistemas dulceacuícolas, incluyendo lagunas, ríos y pequeños arroyos en Argentina, Brasil, Paraguay, y Uruguay. Alcanza un tamaño máximo de 25 centímetros y en algunos sitios pueden llegar a ser los principales peces detritívoros (Vari, 1992). No es una especie de interés comercial y su uso se limita a carnada en la pesca artesanal y deportiva. En cuanto a estudios sobre la fauna parasitaria de C. platanus se cuenta con relativamente pocos trabajos, destacándose los estudios sobre digeneos con las descripciones de nuevas especies. Así, Saccocoelioides magnus Szidat, 1954 (Haploporidae) es citado como parásito de $C$. platanus (=Curimata platana) en el río Paraná frente a Rosario, Argentina (Szidat, 1954), Saccocoelioides antonioi Lunaschi, 1984 y Saccocoelioides platensis Lunaschi, 1984 en C. platanus (=Curimatorbis platanus) provenientes del Río de la Plata en la Provincia de Buenos Aires, Argentina (Lunaschi, 1984). Posteriormente Lunaschi (1988) cita por primera vez para Argentina a Zonocotyle bicaecata Travassos, 1948 en C. platanus de la Provincia de Buenos Aires.

Debido a que únicamente existen estudios descriptivos sobre digeneos en $C$. platanus, el presente trabajo tiene como objetivo un estudio de las poblaciones de digeneos en $C$. platanus procedentes del tramo inferior del río Uruguay en la zona de influencia de la represa de Salto Grande, analizando su asociación con el sexo y tamaño del hospedador, y las posibles asociaciones entre especies de digeneos que parasiten el mismo órgano.

\section{Materiales y Métodos}

Entre los meses de agosto de 2012 y junio de 2013 se obtuvieron sabalitos $C$. platanus de la zona del puerto de la ciudad de Salto, Uruguay $\left(31^{\circ} 23^{\prime} 9.38^{\prime \prime} S\right.$, $57^{\circ} 58^{\prime} 33.08^{\prime \prime O}$ ) en el tramo inferior del río Uruguay. El río Uruguay convencionalmente es dividido en tres tramos según sus características hidrográficas, presentando una extensión total de $1770 \mathrm{~km}$. El tramo inferior se extiende desde la ciudad de Salto (Departamento de Salto) hasta la de Nueva Palmira (Departamento de Colonia). Los peces fueron colectados por pescadores artesanales con redes (atarraya o tarrafa) quienes los pescan para ser utilizados como carnada. La determinación genérica y específica de los peces se basó en Azpelicueta y Braga (1991). Se obtuvo, el largo estándar, el largo total y el peso de los peces y se colocaron en bolsas de polietileno en forma individual. Con los datos de longitud total y peso se calculó el factor de condición relativo $K_{n}$ según Bechara et al., (1999). En el laboratorio se realizó la apertura y procesamiento de los órganos, cavidades y músculo, siguiendo la metodología propuesta por Eiras et al., (2003). Cada órgano y los músculos se observaron bajo lupa estereoscópica, y el contenido del aparato digestivo fue sedimentado en una copa a modo de facilitar su lectura en cajas de Petri. Los parásitos recolectados se fijaron en formol $5 \%$ por 24 horas y luego fueron conservados en alcohol $70 \%$. Los digeneos hallados fueron determinados hasta el nivel taxonómico más bajo posible siguiendo a Thatcher (2006), Gibson et al., (2002), Jones et al., (2005), y Bray et al., (2008).

Los ejemplares más representativos de cada taxón fueron teñidos y montados en preparados permanentes a fin de observar ciertas estructuras anatómicas de valor taxonómico. Para ello los parásitos fueron deshidratados en una serie de alcoholes, se colorearon con carmín de Semichon, se aclararon con eugenol y se montaron en Bálsamo de Canadá.

Se determinaron los siguientes índices poblacionales parasitarios: prevalencia (porcentajes de peces parasitados), intensidad media ( $\mathrm{n}$ - total de parásitos / $\mathrm{n}$ ㅇ de peces parasitados) y abundancia media ( $n$ o total de parásitos / no de peces examinados) según lo establecido por Bush et al., (1997).

Análisis estadístico. La prevalencia se presenta como un porcentaje conjuntamente con su intervalo de confianza al 95\%. La intensidad media y la abundancia media de infección se expresan junto con sus errores típicos. La distribución de las poblaciones de las distintas especies de digeneos en la muestra de hospedadores se evaluó mediante la razón varianza / media de sus intensidades de infección. La asociación entre la prevalencia de infección y el sexo del hospedador y el tamaño de los mismos fue evaluada mediante la prueba de independencia de Chi cuadrado. La relación entre la intensidad de infección y el sexo del hospedador se analizó por medio del Test $U$ de Mann-Whitney. La correlación entre la intensidad de infección y la longitud corporal, el peso y el factor de condición relativo $K_{n}$ se evaluó mediante el coeficiente de correlación por rangos rs de Spearman (Daniel, 1998). En el caso de las dos especies de digeneos halladas parasitando el mismo hábitat (intestino) se estudió la posible asociación entre sus 
prevalencias de infección por medio de la prueba de independencia de Chi cuadrado y entre sus intensidades de infección por medio del coeficiente de correlación por rangos de Spearman. Las diferencias se consideraron significativas con un $\alpha$ de 0.05 .

\section{Resultados}

Se examinaron un total de 76 ejemplares asignados a la especie $C$. platanus. El largo total y el peso se determinaron para 75 de los 76 peces colectados, correspondiendo la longitud promedio a $16.3 \mathrm{~cm}$ (rango: $12.5-19.0 \mathrm{~cm}$ ), y el peso promedio a $59.2 \mathrm{~g}$ (rango: $37.0-86.5 \mathrm{~g}$ ). El factor de condición corporal relativo tuvo un valor promedio de 1.006 , con un rango de 0.6895 a 1.2489. El sexo del hospedador se determinó en 60 peces (en 16 no se pudo determinar), correspondiendo a 35 machos y 25 hembras. El 69,7\% (53/76) de los $C$. platanus analizados resultaron parasitados por al menos un taxón de digeneos. En total se identificaron tres taxones, Saccocoelioides spp. y Zonocotyle bicaecata en intestino, y metacercarias de Sphincterodiplostomum musculosum en el interior de los ojos.

Saccocoelioides (Fig. 1A) es un género de la familia Haploporidae que se caracteriza por tener cuerpo fusiforme con pigmentación dispersa, cubierto de espinas y terminación posterior roma. Ventosa oral terminal y ventosa ventral del mismo tamaño que la oral. Dos ciegos en forma de saco relativamente cortos que terminan posteriormente a la mitad del cuerpo. Glándulas vitelinas constituidas por dos grupos distintos de folículos distribuidos en hileras laterales (Overstreet y Curran, 2005).

Zonocotyle bicaecata (Fig. 1B) pertenece a la familia Zonocotylidae, se caracteriza por tener acetábulo subterminal con surcos en el piso, presencia de un testículo, y glándulas vitelinas compactas y subesféricas (Thatcher, 2006).

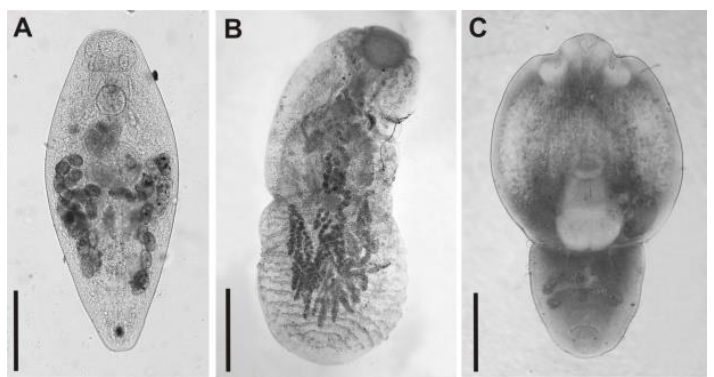

Figura 1. A) Saccocoelioides sp. ejemplar maduro in toto. Escala $=0.4 \mathrm{~mm}$; B) Zonocotyle bicaecata ejemplar maduro in toto teñido. Escala= $1.0 \mathrm{~mm}$; C) Sphincterodiplostomum musculosum metacercaria desenquistada in toto. Escala $=0.7$ $\mathrm{mm}$.

En el caso de $S$. musculosum (Fig. 1C), se corresponde a metacercarias típicas de la familia Diplostomidae y caracterizadas por poseer el cuerpo subdividido en dos partes, anterior oval y cóncava ventralmente, y parte posterior ovoide y más estrecha. La parte anterior se compone de tres lóbulos: el lóbulo medio que está ocupado por la ventosa oral y los lóbulos laterales donde se ubican las pseudoventosas, el acetábulo se encuentra por detrás de la mitad de la parte anterior (Zago et al., 2013).

A lo largo del estudio se observó que Saccocoelioides spp. posee mayores valores en sus índices poblacionales que Z. bicaecata y S. musculosum (Tabla 1). En cuanto a las coinfecciones, 10 C. platanus $(13,1 \%)$ estaban parasitados con Saccocoelioides spp. y Z. bicaecata, dos $(2,6 \%)$ con Z. bicaecata y S. musculosum, y ninguno con Saccocoelioides spp. y $S$. musculosum. La razón varianza / media de las intensidades de infección fue de 11.68 para Saccocoelioides spp., de 1.13 para Z. bicaecata y de 3.73 para las metacercarias de $S$. musculosum.

La Tabla 1 presenta también los índices de infección en función del sexo de los hospedadores. No se hallaron diferencias significativas entre sexos ni en la prevalencia ni en la intensidad de infección para ninguno de los taxones analizados (en el caso de $S$. musculosum, por insuficiencia de datos, no se pudo hacer el análisis correspondiente a la intensidad de infección).

La correlación entre intensidad de infección y longitud total de los hospedadores presentó una asociación negativa en el caso de Saccocoelioides spp. ( $r s=-0.229$, z $=-1.97, p=0.0244)$. Por su parte, la intensidad de infección con ese mismo parásito presentó una ligera correlación positiva con el factor de condición relativo $K_{n}(r s=0.192, z=1.65, p=0.0495)$. La intensidad de infección de los otros dos taxones de digeneos parásitos no presentó ninguna correlación significativa con la longitud total, el peso (en este caso tampoco la presentó Saccocoelioides spp.) o el factor de condición relativo de sus hospedadores.

Tanto la prevalencia como la intensidad de infección de los dos digeneos intestinales (Saccocoelioides spp. y Zonocotyle bicaecata) mostraron ser independientes entre las distintas especies.

\section{Discusión}

La longitud total máxima de los peces estudiados (19.0 $\mathrm{cm})$ fue menor que la máxima señalada para esta especie $(25.0 \mathrm{~cm})$, lo cual tal vez indique restricciones alimentarias o térmicas en el hábitat estudiado. No obstante, el factor de condición corporal relativo, $K_{n}$, tuvo un valor de 1.006, muy próximo al esperado (1.0). A lo largo del estudio la prevalencia de digeneos en $C$. platanus fue relativamente alta $(69,7 \%)$, comparada con los resultados obtenidos por Abdalah et al. (2005) en Cyphocharax gilbert (Quoy e Gaimard, 1824) y Vieira et al. (2013) en Cyphocharax nagelii (Steindachner, 1881), destacando que estos dos trabajos son 
de los más relevantes en cuanto a estudios de ecología parasitaria en el género Cyphocharax. Saccocoelioides fue el género de digeneo más prevalente $(43,4 \%)$. Actualmente dos especies de este género son registradas en $C$. platanus en el río Paraná y Río de la Plata: $S$. magnus y $S$. antonioi, ya que $S$. platensis ha sido transferida al género Chalcinotrema (Overstreet $y$ Curran, 2005). Debido a que morfológicamente se observó una alta variabilidad entre los ejemplares de Saccocoelioides, y que la identificación de las especies de este género es compleja, se prefirió no asignar a ninguna especie los ejemplares obtenidos.

Tabla 1: Índices de infección parasitaria de digeneos en machos y hembras de Cyphocharax platanus del río Uruguay: Prev.: Prevalencia (con intervalo de confianza al $95 \%$ cuando corresponde); Int. M: Intensidad media \pm error típico; Ab. M: Abundancia media \pm error típico; N: número de hospedadores examinados.

\begin{tabular}{|c|c|c|c|c|c|c|c|c|c|}
\hline & \multicolumn{3}{|c|}{ Saccocoelioides spp. } & \multicolumn{3}{|c|}{ Z. bicaecata } & \multicolumn{3}{|c|}{ S. musculosum } \\
\hline & Prev. (\%) & Int. M & Ab. M & Prev. (\%) & Int. M & Ab. M & Prev. (\%) & Int. M & Ab. M \\
\hline General & 43.4 & $5.45 \pm$ & $2.37 \pm$ & 28.9 & $1.32 \pm$ & $0.38 \pm$ & 13.2 & $2.80 \pm$ & $0.37 \pm$ \\
\hline \multirow[t]{2}{*}{$(N=76)$} & $(32.3-54.5)$ & 1.20 & 0.60 & $(18.7-39.1)$ & 0.10 & 0.07 & (5.6- & 0.63 & 0.13 \\
\hline & & & & & & & 20.8) & & \\
\hline Machos & 51.4 & $6.28 \pm$ & $3.23 \pm$ & 34.3 & $1.33 \pm$ & $0.46 \pm$ & - & - & - \\
\hline$(\mathrm{N}=35)$ & $(34.8-68.0)$ & 1.68 & 1.01 & $(18.6-50.0)$ & 0.14 & 0.12 & & & \\
\hline Hembras & 52.0 & $5.00 \pm$ & $2.60 \pm$ & 24.0 & $1.33 \pm$ & $0.32 \pm$ & 4.0 & 2.0 & 0.08 \\
\hline$(N=25)$ & $(32.4-71.6)$ & 1.97 & 1.13 & $(7.3-40.7)$ & 0.21 & 0.13 & & & \\
\hline
\end{tabular}

En tanto que Saccocoelioides spp. y S. musculosum presentaron la típica distribución poblacional agregada que caracteriza a las poblaciones de parásitos (varianza > que la media de la intensidad de infección), en el caso de $Z$. bicaecata se observó una distribución prácticamente aleatoria de los especímenes parasitarios en la población de hospedadores (razón varianza / media $=1.13)$. Según Dobson $(1990)$, la dispersión aleatoria constituye un patrón característico de parásitos con patogenicidad moderada o alta, y con una baja capacidad para colonizar hospedadores.

La ausencia de diferencias en los índices de infección de los tres taxones de digeneos con relación al sexo del hospedador podría indicar que machos y hembras de $C$. platanus tienen una similar conducta trófica y son igualmente susceptibles a la infección con formas infectantes de digeneos. Sólo Saccocoelioides spp. presentó diferencias significativas en cuanto a sus índices de infección con respecto al tamaño de los hospedadores, siendo más frecuente en hospedadores de menor tamaño. Tal asociación podría deberse a una conducta alimenticia de los peces jóvenes que favorece la ingestión de cercarias de haplopóridos, las cuales se encuentran libres en la columna de agua o adheridas a algas y otros sustratos (Martin, 1973; Lado et al., 2013).

Zonocotyle bicaecata fue descripto originalmente en el curimátido Steindachnerina elegans (Steindachner, 1875) (=Curimata elegans) por Travassos (1948). Según los registros de esta especie en Argentina y Brasil, se observa que la misma está estrechamente asociada a peces de la familia Curimatidae. En Brasil los registros para Cyphocharax provienen de C. gilbert [=Zonocotyloides haroltravassosi $=$ Z. bicaecata (Jones, 2005)] y Cyphocharax nagelii (Steindachner, 1881) (Abdalah et al., 2005; Fernandes y Kohn, 2001). Para Argentina fue hallada en $C$. gilbert (=Pseudocurimata gilberti), $C$. platanus (=Curimatorbis platanus) y Steindachnerina brevipinna (Eigenmann \& Eigenmann, 1889) (=Pseudocurimata nitens) (Lunaschi, 1988). En curimátidos de Brasil se han reportado prevalencias de $Z$. bicaecata que van del 6,7\% al 33\% (Travassos, 1948; Abdalah et al., 2005). En nuestro estudio la prevalencia en $C$. platanus fue de $28,9 \%$, la cual se encuentra dentro del rango de los trabajos llevados a cabo en Brasil. Si bien su ciclo biológico no ha sido estudiado en su totalidad, Padilha (1978) observa que los huevos poseen una cáscara membranosa que permite que aumenten de tamaño a medida que se desarrolla el miracidio. Los miracidios emergen de los huevos en el útero y salen al exterior a través del atrio genital, poseen fototropismo positivo y sobreviven en el exterior entre $8 \mathrm{y}$ 10 horas.

Las metacercarias de $S$. musculosum tuvieron bajos índices parasitarios: una prevalencia de $13,1 \%$ y una intensidad de infección de 2,80. Los adultos de esta especie parasitan aves (Lunaschi y Drago, 2006), entre ellas Ardea alba, especie de garza muy frecuente en la zona estudiada. Estos datos difieren con los obtenidos 
en C. gilbert por Abdallah et al. (2005) quienes hallan una prevalencia del $40 \%$ y una intensidad de 11,4 , cifras muy superiores a nuestros resultados. En cambio, para curimátidos del género Steindachnerina Fowler, 1906 la prevalencia puede llegar a valores de entre 90 a 100\% (Ceschini et al., 2010; Zago et al., 2013; Brandão et al., 2014).

Los índices de infección de los dos digeneos intestinales (Saccocoelioides spp. y Z. bicaecata) mostraron ser independientes entre sí, lo que sugiere que estos helmintos no interactúan de ninguna manera en su sitio de infección.

Finalmente, los registros de Saccocoelioides spp. y Z. bicaecata corresponden a los primeros para $C$. platanus en el río Uruguay, y el hallazgo de las metacercarias de $S$. musculosum constituye una nueva relación parásito-hospedador.

\section{Agradecimientos}

A los pescadores artesanales de la zona del puerto de la ciudad de Salto por proveernos las muestras. A la Dra. Graciela Fabiano y Lic. Sebastián Serra por proporcionarnos bibliografía para identificar los peces. Este trabajo fue financiado parcialmente a través del proyecto: Aspectos sanitarios de la fauna íctica de la región de Salto Grande - Convenio específico entre la Comisión Técnica Mixta de Salto Grande (CTM) y la Regional Norte (Universidad de la República). A los dos revisores anónimos quienes contribuyeron a mejorar el trabajo.

\section{Bibliografía}

Abdalah VD, Azevedo RK, Luque JL. 2005. Community ecology of metazoan parasites of Cyphocharax gilbert (Quoy e Gaimard, 1824) (Characiformes: Curimatidae) from Guandu river, State of Rio de Janeiro, Brazil. Rev. Bras. Parasitol. Vet. 14 (4): 154-159.

Azpelicueta MM \& Braga L. 1991. Los Curimátidos en Argentina. Fauna de Agua Dulce de la República Argentina. La Plata, PROFADU-CONICET. 40 (1): 1-53.

Bechara JA, Sánchez S, Roux JP, Terraes JC, Quintana CF. 1999. Variaciones del factor de condición relativo de la ictiofauna del río Paraná aguas debajo de la represa de Yaciretá, Argentina. Rev. Ictiol. 7 ( ${ }^{\circ}$ Especial): 75-89.

Brandão H, Yamada FH, Toledo GM, Acosta AA, Carvalho ED, Silva RJ. 2014. Parasitism by Sphincterodiplostomum musculosum (Digenea, Diplostomidae) metacercariae in the eyes of Steindachnerina insculpta (Characiformes, Curimatidae). Rev. Bras. Parasitol. Vet. 23 (2): 144-149.

Bray RA, Gibson DI, Jones A. 2008. Keys to the Trematoda. London, CAB International. Vol. III. 824 pp.
Bush AO, Lafferty KD, Lotz JM, Shostak AW. 1997. Parasitology meets ecology on its own term: Margolis et al. Revised. J. Parasitol. 83 (4): 575-583.

Ceschini TL, Takemoto, RM, Yamada FH, Moreira LHA, Pavanelli, GC. 2010. Endoparasites of Steindachinerina brevipinna (Eigenmann and Eigenmann, 1889), collected in the tributaries Corvo and Guairacá of Paranapanema river, Paraná State, Brazil. Acta Sci. Biol. Sci. 32 (2): 125-130.

Corrêa F \& Noguez Piedras SR. 2008. Alimentação de Cyphocharax voga (Hensel, 1869) (Characiformes, Curimatidae) no arroio Corrientes, Pelotas, Rio Grande do Sul, Brasil. Biotemas 21: 117-122.

Daniel WW. 1998. Bioestadística. Base para el análisis de las ciências de la salud. UTEHA NORIEGA EDITORES, México, 878 pp.

Dobson AP. 1990. Models of multi-species parasite-host communities. In: Esch GW, Bush AO, Aho J. Parasite communities: patterns and process. New York: Chapman \& Hall; 1990. Pp. 261-288.

Dutra GM, Penido IdS, Mello GCGd, Pessali, TC. 2016. Two new species of Cyphocharax (Teleostei: Characiformes: Curimatidae) from headwaters of the Jequitinhonha and São Francisco river basins, Minas Gerais, Brazil. Zootaxa 4103 (2): 154-164.

Eiras JC, Takemoto RM, Pavanelli GC. 2003. Métodos de estudio y técnicas laboratoriales en parasitología de peces. Editorial Acribia, Zaragoza, España. 142 p.

Fernandes B \& Kohn A. 2001. On some trematodes parasites of fishes from Paraná. River. Braz. J. Biol. 61 (3): 461-466.

Gibson D, Jones A, Bray R. 2002. Keys to the Trematoda. London, CAB International. Vol. I. 521 pp.

Jones A. 2005. Family Zonocotylidae Yamaguti, (1963). En: A. Jones, RA Bray and DI Gibson. Keys to the Trematoda. London, CAB International. Vol. II. pp. 349-351.

Jones A, Bray R, Gibson D. 2005. Keys to the Trematoda. London, CAB International. Vol. II. 745 p.

Lado P, Carnevia D, Perretta A, Castro O. 2013. Heleobia conexa (Mollusca, Cochliopidae) y Mugil platanus (Osteichthyes, Mugilidae), hospedador intermediario y definitivo de Dicrogaster fastigatus (Trematoda, Haploporidae) en Uruguay. Rev. Arg. Parasitol. 2 (1): 20-25.

Lunaschi LI. 1984. Helmintos parásitos de peces de agua dulce de la Argentina. I. Tres nuevas especies del género Saccocoelioides Szidat, 1954 (Trematoda: Haploporidae). Neotropica 30: 31-42.

Lunaschi LI. 1988. Helmintos parásitos de peces de la Argentina. VIII. Zonocotyle bicaecata Travassos, 1948 (Trematoda, Zonocotylidae). Neotropica 34: 83-88.

Lunaschi LI \& Drago FB. 2006. First report of adult specimens of Spincterodiplostomum musculosum (Digenea, Diplostomidae). Parasitol. Int. 55: 7-10.

Martin WE. 1973. Life history of Saccocoelioides pearsoni $\mathrm{n}$. $\mathrm{sp}$. and the description of Lecithobotrys sprenti $\mathrm{n}$. $\mathrm{sp}$. (Trematoda: Haploporidae). Trans. Amer. Microsc. Soc. 92 (1): 80-95.

Melo BF \& Vari RP. 2014. New species of Cyphocharax (Characiformes: Curimatidae) from the upper rio Negro, Amazon basin. Neotrop. Ichthyol. 12: 327-332. 
1914. In: Jones A, Bray RA \& Gibson DI (Eds). Keys to the Trematoda. London, CAB International. Vol. II. pp. 129-165.

Padilha TN. 1978. Caracterização da família Zonocotylidae com redescrição de Zonocotyle bicaecata Travassos, 1948 e descrição de um novo gênero (Trematoda, Digenea). Rev. Bras. Biol. 38: 415-429.

Szidat L. 1954. Tremátodes nuevos de peces de agua dulce de la República Argentina e intento para aclarar su carácter marino. Rev. Inst. Nac. Inves. Cienc. Nat. Argen. Bernadino Rivadavia Zool. 3(1): 1-85.

Thatcher VE. 2006. Aquatic Biodiversity in Latin America. Vol. 1: Amazon fish parasites (2da. Ed.), Pensoft Publishers, SofiaMoscow. $487 \mathrm{pp}$

Travassos L. 1948. Contribuição ao conhecimento dos helmintos dos peixes d'água doce do Brasil. I. Zonocotyle bicaecata n. g. n. sp. (Trematoda, Aspidogastridae). Mem. Inst. Oswaldo Cruz 45 (2): 513-516.

Vari RP. 1992. Systematics of the Neotropical characiform genus Cyphocharax Fowler (Pisces, Ostariophysi). Smithson. Contrib. Zool. 529: 1-137.

Vari RP. 2003. Family Curimatidae. Pp. 51-64. In: Reis RE, SO Kullander, CJ Ferraris Jr. (Eds.). Check list of the freshwater fishes of South and Central America. Porto Alegre, Edipucrs. pp. 1-729.

Vari RP, Sidlauskas BL, Le Bail PY. 2012. New species of Cyphocharax (Ostariophysi: Characiformes: Curimatidae) from Suriname and French Guiana and a discussion of curimatid diversity on the Guiana Shield. Cybium 36: 63-69.

Vieira DHMD, Caramello LE, Abadallah VD, Silva RJ, Azevedo RK. 2013. Community ecology of metazoan parasites of the sairú Cyphocharax nagelii from the Peixe River. Rev. Bras. Parasitol. Vet. 22 (4): 611-615.

Wosiacki WB \& Miranda DPS. 2013. Description of a new small species of the genus Cyphocharax (Characiformes: Curimatidae) from the lower Amazon basin. Copeia 627-633.

Zago AC, Franceschini L, Ramos IP, Zica EOP, Wunderlich AC, Carvalho ED, da Silva RJ. 2013. Sphincterodiplostomum musculosum (Digenea, Diplostomidae) infecting Steindachnerina insculpta (Characiformes, Curimatidae) in the Chavantes Reservoir, Southeastern Brazil. Rev. Bras. Parasitol. Vet. 22 (1): 98-103. 